\title{
A Synthesis of Heteroaromatic Analogues of 1-Methyl-1,2,3,4- tetrahydroisoquinoline Using the Pummerer-Type Cyclization Reaction: Observation of Tandem Cyclization Reaction
}

\author{
Yoshie Horiguchi, ${ }^{*}$ Keita Ogawa, Toshiaki Saitoh, and Takehiro Sano \\ Showa Pharmaceutical University; 3-3165 Higashi-tamagawagakuen, Machida, Tokyo 194-8543, Japan. \\ Received September 9, 2003; accepted November 18, 2003
}

\begin{abstract}
The sulfoxides $7 \mathrm{~b}$ and $7 \mathrm{~d}$ carrying thiophene or benzothiophene as heteroaromatic nucleophiles, when treated with trifluoroacetic anhydride at room temperature (Pummerer reaction), underwent an intramolecular alkylation in an exclusive manner to yield 4,5,6,7-tetrahydro-7-methyl-4-phenylsulfanylthieno[2,3-c]pyridine-6carbaldehyde (10) and the corresponding benzothiophene derivative (12b) in high yields, respectively. Thus, this route provides biologically interesting nitrogen heterocycles (1b) and (2b). On the other hand, the sulfoxide (7c) carrying benzofuran as a nucleophile on reaction with TFAA yielded not only the Pummerer-type cyclization product (12a), but also the diastereoisomeric tandem cyclization products (13) and (14) having a noble 11-aza-2oxa-7-thiatricyclo[4.3.3.0 ${ }^{1,5}$ dodecane ring system $(B)$. The formation of these products can be readily rationalized by the intervention of the oxonium ion intermediate (21).
\end{abstract}

Key words pummerer reaction; thieno[2,3-c]pyridine; benzothieno[2,3-c]pyridine; benzofuro[2,3-c]pyridine; 11-aza-2-oxa-7thiatricyclo[4.3.3. $\left.0^{1,5}\right]$ dodecane

In a series of papers we reported the synthesis of $1,2,3,4-$ tetrahydroisoquinolines, ${ }^{1-6)} 1,2,3,4$-tetrahydroquinolines, ${ }^{7}$ 2,3,4,5-tetrahydro- $1 H$-3-benzazepines, ${ }^{8)}$ 2-quinolones ${ }^{9)}$ using an aromatic cyclization of the sulfonium ion in situ formed from a sulfinyl purecursor (Pummerer reaction). The aromatic cyclization in the reaction using trifluoroacetic anhydride (TFAA) as a sole reagent (method A) smoothly proceeded at room temperature when the reactive center of the cyclizing benzene ring was activated by the electron-donating substituent. Furthermore, we found that the Pummerer substrate which lacks an electron-donating substituent on the cyclizing benzene ring, requires boron trifluoride diethyl etherate $\left(\mathrm{BF}_{3} \cdot \mathrm{Et}_{2} \mathrm{O}\right)$ as an additive reagent (method $\left.\mathrm{B}\right)$ to induce the aromatic cyclization. ${ }^{2,3)}$ Thus, the investigations demonstrated that the Pummerer-type cyclization reaction is highly effective and widely applicable for the construction of aromatic condensed nitrogen heterocycles. ${ }^{10)}$

In order to expand the utility of this methodology, we designed a synthesis of nitrogen heterocycles possessing furo(1a), thieno- (1b), benzofuro- (2a) and benzothieno[2,3c]pyridine (2b), which bear a methyl group on the nitrogencontaining ring. The compounds with the ring system are expected to have some biological activities related to parkinsonism because of their structural resemblance to 1-methyl1,2,3,4-tetrahydroisoquinoline (3), endogenous amine present in the human brain, ${ }^{11)}$ which is considered to play an important role in the prevention of the onset of parkinsonism induced by the selective dopaminergic neurotoxin, 1-methyl4-phenylpyridinium ion $\left(\mathrm{MPP}^{+}\right)$, and by other endogenous neurotoxins, 1,2,3,4-tetrahydroisoquinoline and 1-benzyl1,2,3,4-tetrahydroisoquinoline. ${ }^{12-14)}$

\section{Results and Discussion}

$N$-Formyl sulfoxides $(\mathbf{7 a}-\mathbf{d})$, substrates of the Pummerer reaction, were prepared from 2-acetylfuran (4a), 2-acetylthiophene (4b), 2-acetylbenzofuran (4c), and 2-acetylbenzothiophene (4d) in three steps (Chart 2). Condensation of $\mathbf{4}$ with 2-phenylsulfanylethylamine in titanium(IV) isopropoxide followed by $\mathrm{NaBH}_{4}$ reduction of the resulting imines afforded the amines $\mathbf{5}$ in good yields. Formylation of $\mathbf{5}$ and then oxidation of the resulting $N$-formates $\mathbf{6}$ with sodium metaperiodate produced the sulfoxides 7 in high yields, though the sulfones $\mathbf{8}$, in some cases, were produced as a byproduct.

Pummerer Reaction of the Sulfoxides $7 \mathbf{a}, \mathbf{b}$ The sul-
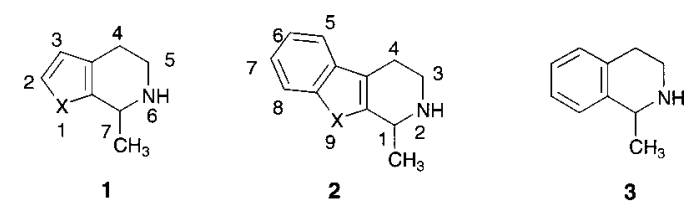

$\mathrm{a}: \mathrm{X}=\mathrm{O}, \mathrm{b}: \mathrm{X}=\mathrm{S}$

a: $X=O, b: X=S$

Chart 1

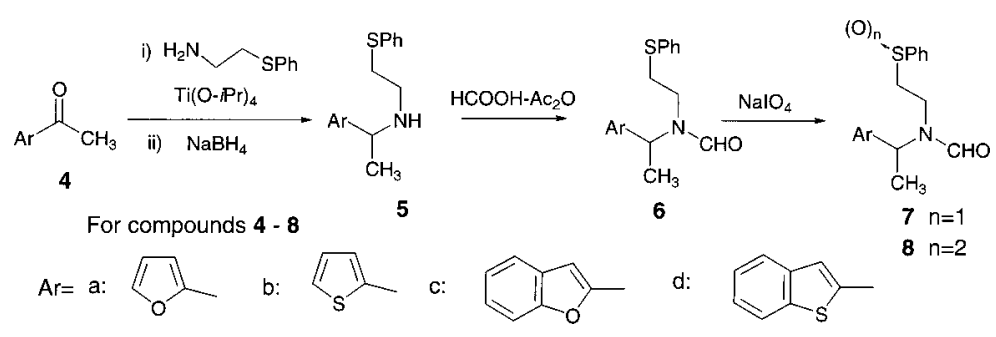

Chart 2 


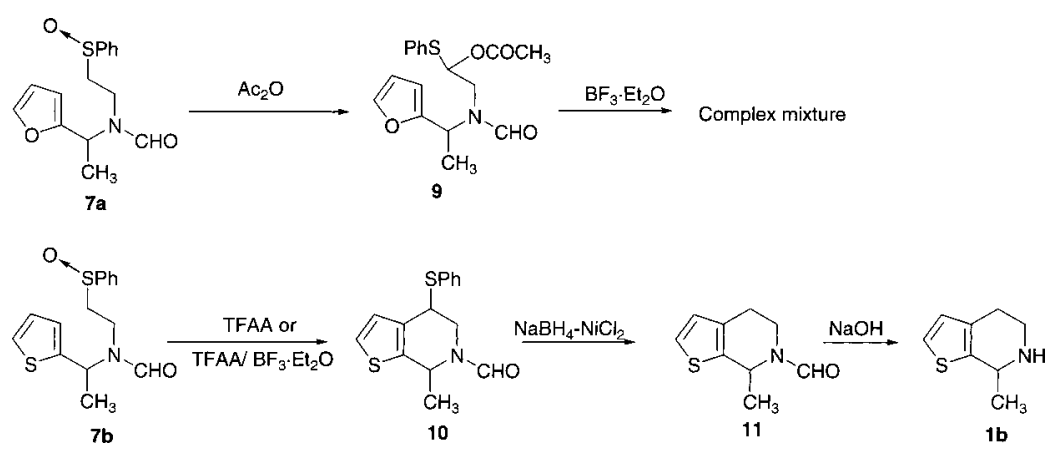

Chart 3

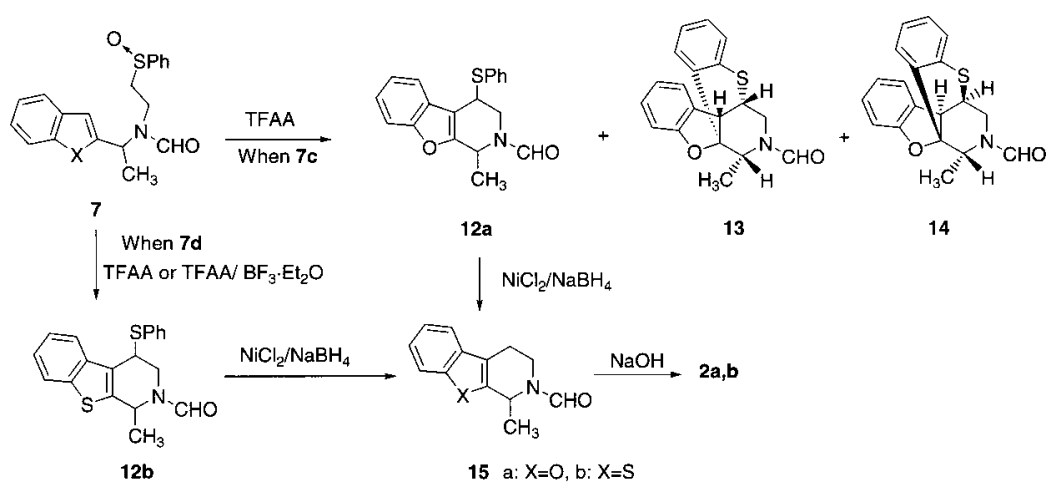

Chart 4

foxide 7a possessing a furan ring as a nucleophilic aromatic ring, on treatment with trifluoroacetic anhydride (TFAA) in benzene at room temperature for $70 \mathrm{~min}$, caused an extensive decomposition to yield no characterizable products. Even a reaction under more mild conditions $\left(0^{\circ} \mathrm{C}, 10 \mathrm{~min}\right)$ merely produced deep colored compounds. These facts suggest that the furan ring is highly vulnerable to trifluoroacetic acid (TFA) generated in the reaction. The treatment of $7 \mathbf{a}$ with acetic anhydride at $80^{\circ} \mathrm{C}$ for $14 \mathrm{~d}$ slowly induced a Pummerer rearrangement to give the $\alpha$-acetoxyl sulfide 9 in $77 \%$ yield. Although this product is considered to be an intermediate of the Pummerer-type cyclization reaction, any attempt leading to the expected cyclization under acidic conditions was failed. For example, the reaction of 9 with $\mathrm{BF}_{3} \cdot \mathrm{Et}_{2} \mathrm{O}$ in benzene at room temperature gave no characterizable products.

On the other hand the sulfoxide $\mathbf{7 b}$ having thiophene ring, when reacted with TFAA in benzene at room temperature for $6 \mathrm{~h}$ (method A), underwent the expected cyclization to produce 4,5,6,7-tetrahydro-7-methyl-4-phenylsulfanylthieno$[2,3-c]$ pyridine-6-carbaldehyde (10). This cyclization, on sequential treatment of $\mathbf{7 b}$ using TFAA and $\mathrm{BF}_{3} \cdot \mathrm{Et}_{2} \mathrm{O}$ (method $\mathrm{B})$, occurred more rapidly $(1 \mathrm{~h})$ to give $\mathbf{1 0}$ in an excellent yield (93\%). Reductive removal of the phenylsulfanyl group of 10 with $\mathrm{NiCl}_{2}-\mathrm{NaBH}_{4}$ in THF produced 4,5,6,7-tetrahydro-7-methylthieno[2,3-c]pyridine-6-carbaldehyde (11) in $61 \%$ yield. Hydrolysis of 11 with $\mathrm{NaOH}$ solution gave the desired product, 4,5,6,7-tetrahydro-7-methylthieno[2,3-c]pyridine (1) $\mathbf{1 b}$ in $79 \%$ yield.

Pummerer Reaction of the Sulfoxides $7 \mathrm{c}$ and $7 \mathrm{~d}$ Treatment of the sulfoxide 7c having benzofuran ring with TFAA in benzene at room temperature for $1.5 \mathrm{~h}($ method A) yielded three products $\mathbf{1 2 a}, \mathbf{1 3}$, and 14 in yields of $36 \%$, $35 \%$, and $16 \%$, respectively (Chart 4). The use of $\mathrm{BF}_{3} \cdot \mathrm{Et}_{2} \mathrm{O}$ as an additional reagent (method B) facilitated the reaction to give the same products $12 \mathrm{a}(30 \%), 13(39 \%)$, and $14(27 \%)$. In contrast the sulfoxide $\mathbf{7 d}$ having benzothiophene ring, on the reaction by either the method A or B, gave 12b in $94 \%$, $99 \%$ yields, respectively.

The structures of 12a and 12b as the expected 4-phenylsulfanyl benzofuro and benzothieno[2,3-c]pyridine derivatives were confirmed by their spectral data and the following chemical transformations. Reductive removal of the phenylthio group of $\mathbf{1 2 a}$ and $\mathbf{1 2} \mathbf{b}$ with $\mathrm{NaBH}_{4}-\mathrm{NiCl}_{2}$ in EtOH produced 15a and $\mathbf{1 5 b}$ in $77 \%$ and $71 \%$ yields, respectively. Deprotection of the $N$-formyl group of $\mathbf{1 5 a}$ and $\mathbf{1 5 b}$ by alkaline hydrolysis yielded 1,2,3,4-tetrahydro-1-methylbenzo[4,5]furo- (2a) and 1,2,3,4-tetrahydro-1-methylbenzo$[4,5]$ thieno[2,3-c]pyridine (2b) in $98 \%$ and $83 \%$ yields, respectively.

Structures of Tandem Cyclization Products 13 and 14 The compounds $\mathbf{1 3}$ and $\mathbf{1 4}$ were identified as the products formed by two successive intramolecular alkylation reactions (tandem cyclization products) as follow. Their respective mass spectrum gave the same molecular peak at $\mathrm{m} / \mathrm{z} 323$ corresponding to the formula $\mathrm{C}_{19} \mathrm{H}_{17} \mathrm{O}_{2} \mathrm{NS}$, which is identical with that of 12a. Their UV and NMR spectral data are very similar each other, but they are significantly different with those of 12a; the facts indicating that the products 13 and $\mathbf{1 4}$ have a different skeletal ring system with that of 12a. The ${ }^{1} \mathrm{H}$ - and ${ }^{13} \mathrm{C}-\mathrm{NMR}$ spectra of $\mathbf{1 3}$ and $\mathbf{1 4}$ exhibited very complicated signals, thus suggesting that they are present in $\mathrm{CDCl}_{3}$ as a mixture of two rotational isomers of $\mathrm{N}-\mathrm{CO}$ bond. Therefore, accurate assignments of the NMR spectra were 
difficult. Fortunately, we were able to clarify their structures including the stereochemistry by the spectral analysis of the $\mathrm{N}$-deformyl derivatives $\mathbf{1 6}$ and $\mathbf{1 7}$ which were prepared by alkaline hydrolysis of $\mathbf{1 3}$ and $\mathbf{1 4}$ in $96 \%$ and $85 \%$ yields, respectively (Chart 5).

The gross structures of $\mathbf{1 6}$ and $\mathbf{1 7}$ were deduced from detailed analysis of the ${ }^{1} \mathrm{H}$ - and ${ }^{13} \mathrm{C}$-NMR data aided by two-dimensional (2D) NMR experiments, $\mathrm{H}-\mathrm{H}$ correlation spectroscopy (COSY), C-H COSY and heteronuclear multiple bond correlation (HMBC). Their ${ }^{1} \mathrm{H}$ - and ${ }^{13} \mathrm{C}-\mathrm{NMR}$ data of $\mathbf{1 6}$ and $\mathbf{1 7}$ indicated that they possessed a same skeleton with twelve aromatic carbons, one quarternary carbon, three methines, one methylene, and one methyl group. All protons and carbons were unambiguously assigned from the 2DNMR spectra analysis as shown in Table 1. HMBC correlations of 16 as shown in Fig. $1, \mathrm{H}-9$ to $\mathrm{C}-7\left(\delta_{\mathrm{C}} 129.6\right)$, to

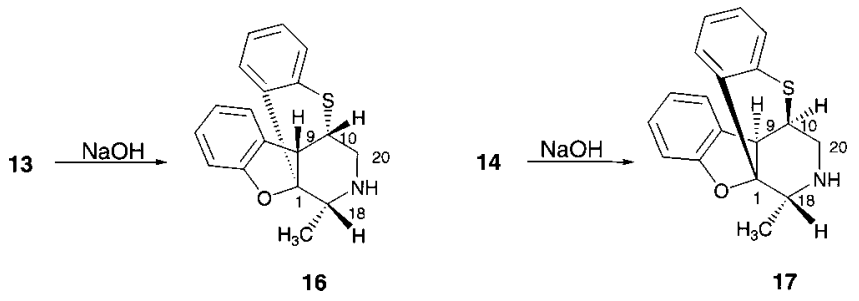

Chart 5

Table $1 .{ }^{1} \mathrm{H}$ - and ${ }^{13} \mathrm{C}$-NMR Chemical Shifts $(\delta, \mathrm{ppm})$ of Products 16 and 17

\begin{tabular}{|c|c|c|c|c|}
\hline \multirow{2}{*}{ Position } & \multicolumn{2}{|l|}{16} & \multicolumn{2}{|l|}{17} \\
\hline & ${ }^{1} \mathrm{H}$ & ${ }^{13} \mathrm{C}$ & ${ }^{1} \mathrm{H}$ & ${ }^{13} \mathrm{C}$ \\
\hline 1 & - & 98.4 & - & 95.1 \\
\hline 3 & - & 157.6 & - & 158.8 \\
\hline 4 & $7.17(\mathrm{dd}, J=8,1 \mathrm{~Hz})$ & 124.7 & $7.12(\mathrm{~d}, J=8 \mathrm{~Hz})$ & 124.7 \\
\hline 5 & $7.09(\mathrm{t}, J=8 \mathrm{~Hz})$ & 128.8 & $7.09(\mathrm{t}, J=8 \mathrm{~Hz})$ & 128.9 \\
\hline 6 & $7.06(\mathrm{t}, J=8 \mathrm{~Hz})$ & 126.7 & $7.06(\mathrm{td}, J=8,1 \mathrm{~Hz})$ & 126.6 \\
\hline 7 & $7.45(\mathrm{~d}, J=8 \mathrm{~Hz})$ & 129.6 & $7.43(\mathrm{dd}, J=8,1 \mathrm{~Hz})$ & 129.4 \\
\hline 8 & - & 133.6 & - & 134.2 \\
\hline 9 & $4.58(\mathrm{~s})$ & 46.4 & $4.47(\mathrm{~s})$ & 47.1 \\
\hline 10 & $3.55(\mathrm{dd}, J=11,8 \mathrm{~Hz})$ & 46.8 & $3.53(\mathrm{dd}, J=12,8 \mathrm{~Hz})$ & 50.3 \\
\hline 12 & - & 129.9 & - & 129.7 \\
\hline 13 & $7.19(\mathrm{~d}, J=8 \mathrm{~Hz})$ & 129.5 & $7.22(\mathrm{dd}, J=8,1 \mathrm{~Hz})$ & 129.9 \\
\hline 14 & $6.78(\mathrm{td}, J=8,1 \mathrm{~Hz})$ & 120.7 & $6.76(\mathrm{td}, J=8,1 \mathrm{~Hz})$ & 120.6 \\
\hline 15 & $7.14(\mathrm{td}, J=8,1 \mathrm{~Hz})$ & 126.2 & $7.16(\mathrm{td}, J=8,1 \mathrm{~Hz})$ & 126.3 \\
\hline 16 & $6.80(\mathrm{~d}, J=8 \mathrm{~Hz})$ & 110.0 & $6.82(\mathrm{~d}, J=8 \mathrm{~Hz})$ & 109.2 \\
\hline 17 & - & 137.5 & - & 137.6 \\
\hline 18 & $3.59(\mathrm{q}, J=7 \mathrm{~Hz})$ & 60.1 & $3.25(\mathrm{q}, J=7 \mathrm{~Hz})$ & 62.3 \\
\hline \multirow[t]{2}{*}{20} & $3.25(\mathrm{dd}, J=10,11 \mathrm{~Hz})$ & 46.5 & $3.26(\mathrm{dd}, J=10,11 \mathrm{~Hz})$ & 46.5 \\
\hline & $3.42(\mathrm{dd}, J=10,8 \mathrm{~Hz})$ & & $3.41(\mathrm{dd}, J=10,8 \mathrm{~Hz})$ & \\
\hline 21 & $1.18(\mathrm{~d}, J=7 \mathrm{~Hz})$ & 20.3 & $1.11(\mathrm{~d}, J=7 \mathrm{~Hz})$ & 13.4 \\
\hline
\end{tabular}
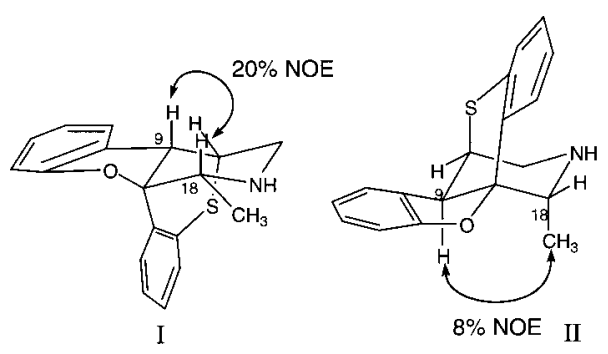

C-3 $\left(\delta_{\mathrm{C}} 157.6\right)$, to $\mathrm{C}-17\left(\delta_{\mathrm{C}} 137.5\right)$, and to $\mathrm{C}-18\left(\delta_{\mathrm{C}} 60.1\right)$, $\mathrm{H}-10$ to $\mathrm{C}-1\left(\delta_{\mathrm{C}} 98.4\right)$, and to $\mathrm{C}-8\left(\delta_{\mathrm{C}} 133.6\right), \mathrm{H}-6$ to $\mathrm{C}-8, \mathrm{H}-$ 5 to $\mathrm{C}-3, \mathrm{H}-14$ to $\mathrm{C} 12\left(\delta_{\mathrm{C}} 129.9\right)$, and $\mathrm{H}-16$ to $\mathrm{C}-12$ indicated that 16 is not a derivative of furo[2,3-c]pyridine skeleton but a derivative of 19-aza-2-oxa-11-thiapentacyclo[8.7.3.0 $\left.0^{1,9} \cdot 0^{3,8} \cdot 0^{12,17}\right]$ icosa-3,5,7,12,14,16-hexaene skeleton (A). This ring system can be constructed by $\mathrm{C}-\mathrm{C}$ bonding between the 9a-carbon of benzofuro[2,3-c]pyridine and the ortho-carbon of 4-phenylsulfanyl group. The other product $\mathbf{1 7}$ also exhibited HMBC correlations similar to those of $\mathbf{1 6 .}$ Thus, the compounds $\mathbf{1 6}$ and $\mathbf{1 7}$ are assigned as stereoisomers possessing the skeleton of a unique tricyclic bridged ring system including nitrogen, oxygen, and sulfur atoms (11-aza-2-oxa-7-thiatricyclo[4.3.3.0 $0^{1,5}$ dodecane B).

The structures of $\mathbf{1 6}$ and $\mathbf{1 7}$ have four chiral centers including two bridgehead carbons. Therefore, four stereostructures (I-IV) as racemate are able to be deduced as shown in Fig. 2. Differentiation between the stereo-structures was achieved by nuclear Overhauser effect (NOE) observations. The diastereomer 16 exhibited 20\% NOE between C9proton and $\mathrm{C} 18$-proton, indicating that these protons are arranged in 1,3-diaxial cis-relationship concerning to the piperidine ring. Thus, the relative stereochemistry of $\mathbf{1 6}$ is elucidated to be $1 R^{*}, 9 S^{*}, 10 S^{*}$, and $18 R^{*}$ as shown in the stereo-structure I. On the other hand, the diastereomer 17 exhibited 8\% NOE between $\mathrm{C} 9-\mathrm{H}$ and $\mathrm{C} 18$-methyl proton, indicating that they are orientating in 1,3-diaxial cis-relationship concerning to the piperidine ring. Thus, the relative stereochemistry of 17 are assigned to be $1 S^{*}, 9 R^{*}, 10 R^{*}$, and $18 R^{*}$ as shown in the stereo-structure II. The other stereo-structures III and IV with a equatorial hydrogen at C-9 to the piperidine ring can be discarded since they are not expected to give such NOEs.

In order to clarify the formation mechanism of the Pummerer products $\mathbf{1 3}$ and $\mathbf{1 4}$, we carried out some experiments. The treatment of $7 \mathrm{c}$ with acetic anhydride at $80^{\circ} \mathrm{C}$ for $9 \mathrm{~d}$

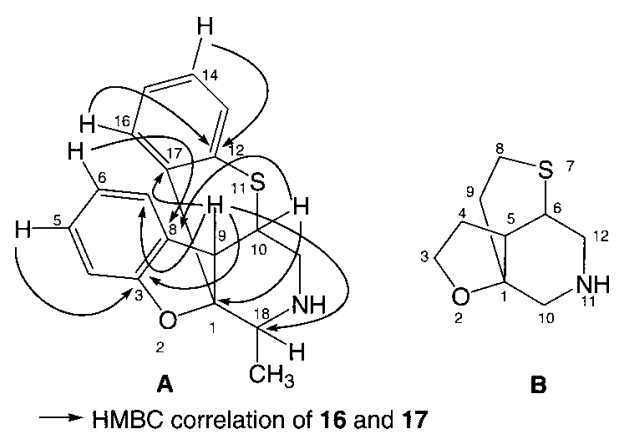

Fig. 1
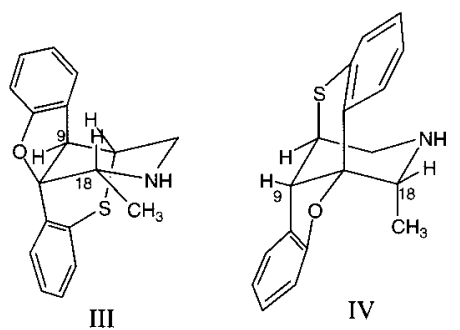

IV

Fig. 2 


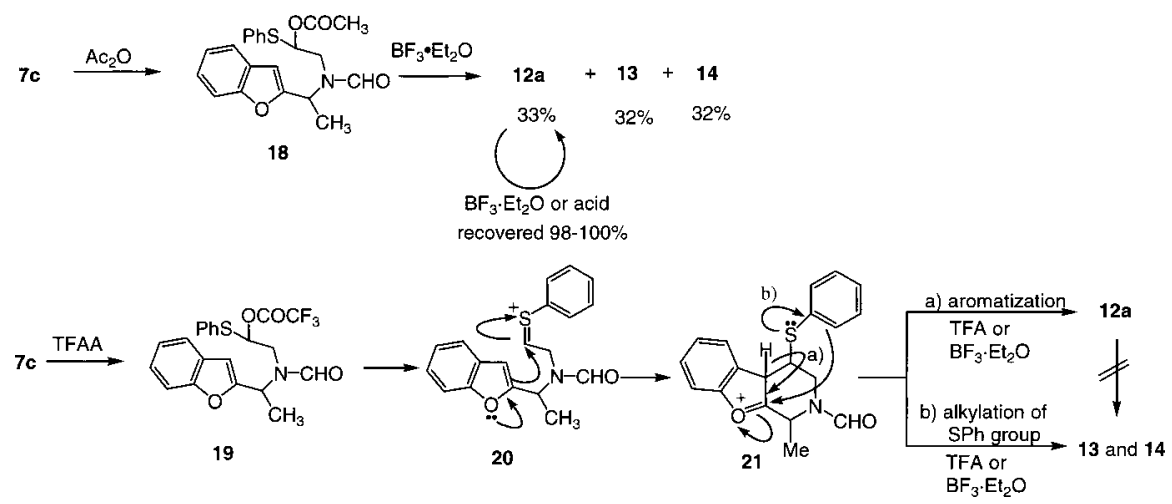

Chart 6

slowly caused the Pummerer-rearrangement reaction to afford the $\alpha$-acetoxyl sulfide $\mathbf{1 8}$ in $87 \%$ yield. The treatment of 18 with $\mathrm{BF}_{3} \cdot \mathrm{Et}_{2} \mathrm{O}$ in benzene at room temperature for $7 \mathrm{~d}$ slowly induced the cyclization to yield 12a (33\%), $13(32 \%)$, and $14(32 \%)$, which were proved to be identical with the products obtained from the TFAA-induced Pummerer reaction of 7c described above. On the other hand, acidic treatment of the Pummerer product 12a with $\mathrm{BF}_{3} \cdot \mathrm{Et}_{2} \mathrm{O}$-TFAA, TFA-TFAA or $p$-TsOH-acetic anhydride did not cause any reaction and the starting material was recovered quantitatively (see experimental).

The formation mechanism of the products 12a, 13, and 14 is rationalized as shown in Chart 6 . The formation of these products can be initiated by the generally accepted mechanism of the Pummerer reaction. The reaction involves two processes. One is a nucleophilic attack of the benzofuran ring on the cationic carbon atom of the sulfonium ion $\mathbf{2 0}$ followed by the aromatization reaction of the formed oxonium ion $\mathbf{2 1}$ leading to $\mathbf{1 2 a}$; the other involves the intramolecular alkylation of the C-9a carbon of $\mathbf{2 1}$ to the ortho-carbon of benzene ring of the phenylsulfanyl group. The two reactions should proceed in a competitive manner via the oxonium cation 21, which, however, on treatment with $\mathrm{BF}_{3} \cdot \mathrm{Et}_{2} \mathrm{O}$ is not generated from 12a. The compound with 11-aza-2-oxa-7-thiatricyclo[4.3.3.0 $\left.0^{1,5}\right]$ dodecane skeleton $(\mathbf{B})$ seems to be hitherto unknown.

\section{Experimental}

Unless otherwise noted, the following procedures were adopted. Melting points were taken on a Yanagimoto SP-M1 hot-stage melting point apparatus and are uncorrected. IR spectra were measured as films for oils and gums, and $\mathrm{KBr}$ disks for solids with a HORIBA FT-710 spectrophotometer, and the values are given in $\mathrm{cm}^{-1}$. NMR spectra were measured on a JEOL JNM- $\alpha$ $500\left({ }^{1} \mathrm{H}-\mathrm{NMR}: 500 \mathrm{MHz},{ }^{13} \mathrm{C}-\mathrm{NMR}\right.$ : $\left.125 \mathrm{MHz}\right)$ or a JEOL JNM-AL300 $\left({ }^{1} \mathrm{H}-\right.$ NMR: $300 \mathrm{MHz},{ }^{13} \mathrm{C}-\mathrm{NMR}$ : $75 \mathrm{MHz}$ ) NMR spectrometer in $\mathrm{CDCl}_{3}$ with tetramethylsilane as an internal standard and the chemical shifts are given in $\delta$ values. Low-resolution electron impact ionization mass spectra (LR-EIMS) were taken on JEOL JMS-AM20 mass spectrometer at $70 \mathrm{eV}$ using direct inlet probe. High-resolution EI-MS (HR-EI-MS) and was taken on a JEOL JMS-D300 mass spectrometer at $70 \mathrm{eV}$ using direct inlet system. FAB-MS spectra were recorded with JEOL-HX100A spectrometer using glycerol as a matrix. Elemental analyses were recorded on a Yanaco CHNcorder MT-3. TLC was performed on Merck precoated silica gel $60 \mathrm{~F}_{254}$ plates. Column chromatography was carried out with Wakogel C-200. Flash chromatography was carried out with silica gel 60 (Merck) or silica gel $60 \mathrm{~N}$ (Kanto). The organic extract from each reaction mixture was washed with brine, dried over anhydrous $\mathrm{Na}_{2} \mathrm{SO}_{4}$, and concentrated in vacuo to dryness.

Preparation of 5a. Typical Procedure A mixture of $4 \mathbf{a}(6.00 \mathrm{~g}, 54.5$ $\mathrm{mmol})$, 2-phenylsulfanylethylamine $(10.03 \mathrm{~g}, 66 \mathrm{mmol})$, and titanium(IV) isopropoxide $(23.10 \mathrm{~g}, 81 \mathrm{mmol})$ was heated at $80^{\circ} \mathrm{C}$ for $3 \mathrm{~h}$ under an argon atmosphere. After cooling, the reaction mixture was diluted with $\mathrm{MeOH}$ $(100 \mathrm{ml})$. To this solution, $\mathrm{NaBH}_{4}(2.48 \mathrm{~g}, 66 \mathrm{mmol})$ was added in small portions under ice-cooling. The reaction mixture was stirred at room temperature for $1 \mathrm{~h}$ and concentrated in vacuo. Water $(c a .40 \mathrm{ml})$ was added to the residue, and the mixture was diluted with $\mathrm{MeOH}(c a .500 \mathrm{ml})$. After removal of precipitated inorganic materials by filtration, the filtrate was concentrated in vacuo. The residue was dissolved in water and acidified by $5 \% \mathrm{HCl}$ solution and the mixture was extracted with $\mathrm{CHCl}_{3}$. Purification by column chromatography (AcOEt : hexane $=1: 2$ ) of the product gave (1-furan-2-yl)ethyl(2-phenylsulfanylethyl)amine (5a) $(13.04 \mathrm{~g}, 97 \%)$ as a yellow oil. IR: 1583 . ${ }^{1} \mathrm{H}-\mathrm{NMR}: 1.37\left(3 \mathrm{H}, \mathrm{d}, J=7 \mathrm{~Hz}, \mathrm{CH}_{3}\right), 2.75\left(2 \mathrm{H}, \mathrm{t}, J=6 \mathrm{~Hz}, \mathrm{SCH}_{2} \mathrm{CH}_{2} \mathrm{~N}\right)$, $3.02\left(2 \mathrm{H}, \mathrm{t}, J=6 \mathrm{~Hz}, \mathrm{SCH}_{2} \mathrm{C}_{2} \mathrm{~N}\right), 3.97\left(1 \mathrm{H}, \mathrm{q}, J=7 \mathrm{~Hz}, \mathrm{C}^{\mathrm{HCH}}\right)_{3}, 6.09(1 \mathrm{H}$, $\mathrm{d}, J=3 \mathrm{~Hz}, \mathrm{Ar}), 6.27(1 \mathrm{H}, \mathrm{dd}, J=2,3 \mathrm{~Hz}, \mathrm{Ar}), 7.1-7.3(6 \mathrm{H}, \mathrm{m}, \mathrm{Ar}) .{ }^{13} \mathrm{C}-$ NMR: $20.3 \quad\left(\mathrm{CHCH}_{3}\right), \quad 34.4 \quad\left(\mathrm{SCH}_{2} \mathrm{CH}_{2} \mathrm{~N}\right), \quad 45.4 \quad\left(\mathrm{SCH}_{2} \mathrm{CH}_{2} \mathrm{~N}\right), \quad 50.9$ $\left(\mathrm{CHCH}_{3}\right), 105.3(\mathrm{ArCH}), 109.8(\mathrm{ArCH}), 126.2(\mathrm{ArCH}), 128.8(\mathrm{ArCH} \times 2)$, $129.8(\mathrm{ArCH} \times 2), 135.6(\mathrm{ArC}), 141.4(\mathrm{ArCH}), 157.5(\mathrm{ArC})$. LR-EI-MS $m / z$ : $247\left(\mathrm{M}^{+}\right)$. HR-EI-MS: Calcd for $\mathrm{C}_{14} \mathrm{H}_{17} \mathrm{NOS}: 247.1031$. Found: 247.1069.

(2-Phenylsulfanylethyl)-(1-thiophen-2-ylethyl)amine (5b) $(7.70 \mathrm{~g}, 81 \%)$ was obtained from 2-acetylthiophene $(\mathbf{4 b})(4.51 \mathrm{~g}, 36 \mathrm{mmol})$ after purification by column chromatography (AcOEt: hexane $=1: 1$ ) as a colorless oil. IR: $1583 .{ }^{1} \mathrm{H}-\mathrm{NMR}: 1.45\left(3 \mathrm{H}, \mathrm{d}, J=7 \mathrm{~Hz}, \mathrm{CH}_{3}\right), 2.8-3.1(4 \mathrm{H}, \mathrm{m}$, $\left.\mathrm{SC}_{2} \mathrm{C}_{2} \mathrm{~N}\right), 4.08\left(1 \mathrm{H}, \mathrm{q}, J=7 \mathrm{~Hz}, \mathrm{CHCH}_{3}\right), 6.9-7.3(8 \mathrm{H}, \mathrm{m}, \mathrm{Ar})$. LR-EIMS $m / z$ : $263\left(\mathrm{M}^{+}\right)$. HR-EI-MS: Calcd for $\mathrm{C}_{14} \mathrm{H}_{17} \mathrm{NS}_{2}: 263.0802$. Found: 263.0806 .

(1-Benzofuran-2-ylethyl)-(2-phenylsulfanylethyl)amine (5c) $(5.37 \mathrm{~g}, 88 \%)$ was obtained from 2-acetylbenzofuran $(4 \mathrm{c})(3.01 \mathrm{~g}, 18.8 \mathrm{mmol})$ after purification by column chromatography (AcOEt: hexane $=1: 1$ ) as a yellow oil. IR: 1583. ${ }^{1} \mathrm{H}-\mathrm{NMR}: 1.59\left(3 \mathrm{H}, \mathrm{d}, J=6 \mathrm{~Hz}, \mathrm{CH}_{3}\right), 2.79(2 \mathrm{H}, \mathrm{t}, J=6 \mathrm{~Hz}$, $\left.\mathrm{SCH}_{2} \mathrm{CH}_{2} \mathrm{~N}\right), 3.04\left(2 \mathrm{H}, \mathrm{t}, J=6 \mathrm{~Hz}, \mathrm{SCH}_{2} \mathrm{CH}_{2} \mathrm{~N}\right), 3.97(1 \mathrm{H}, \mathrm{q}, J=6 \mathrm{~Hz}$, $\left.\mathrm{CHCH}_{3}\right), 6.47(1 \mathrm{H}, \mathrm{s}, \mathrm{Ar}), 7.1-7.5(9 \mathrm{H}, \mathrm{m}, \mathrm{Ar}) .{ }^{13} \mathrm{C}-\mathrm{NMR}: 20.5\left(\mathrm{CHCH}_{3}\right)$, $34.5\left(\mathrm{SCH}_{2} \mathrm{CH}_{2} \mathrm{~N}\right), 45.4\left(\mathrm{SCH}_{2} \mathrm{CH}_{2} \mathrm{~N}\right), 51.4\left(\mathrm{CHCH}_{3}\right), 102.4(\mathrm{ArCH}), 111.1$ $(\mathrm{ArCH}), 120.7(\mathrm{ArCH}), 122.6(\mathrm{ArCH}), 123.7(\mathrm{ArCH}), 126.3(\mathrm{ArCH}), 128.3$ $(\mathrm{ArC}), 128.8(\mathrm{ArCH} \times 2), 130.0(\mathrm{ArCH} \times 2), 135.3(\mathrm{ArC}), 154.7(\mathrm{ArC})$, 160.2 (ArC). LR-EI-MS m/z: $297\left(\mathrm{M}^{+}\right)$. HR-EI-MS: Calcd for $\mathrm{C}_{18} \mathrm{H}_{19} \mathrm{NOS}$ : 297.1188. Found: 297.1218.

Formylation of 5a. Typical Procedure A solution of formic-acetic anhydride prepared from formic acid $(74.6 \mathrm{ml}, 1.96 \mathrm{~mol})$ and acetic anhydride $(46 \mathrm{ml}, 0.49 \mathrm{~mol})$, was added to $5 \mathbf{a}(12.02 \mathrm{~g}, 49 \mathrm{mmol})$ at $0{ }^{\circ} \mathrm{C}$ in one portion and then the mixture was heated at $70{ }^{\circ} \mathrm{C}$ for $1 \mathrm{~h}$. The reaction mixture was concentrated in vacuo and extracted with $\mathrm{CHCl}_{3}$. The residue was chromatographed (AcOEt: hexane $=1: 2$ ) to give $N$-(1-furan-2-ylethyl)- $N$ (2-phenylsulfanylethyl)formamide (6a) $(12.97 \mathrm{~g}, 97 \%)$ as a yellow oil. IR: 1672. ${ }^{1} \mathrm{H}-\mathrm{NMR}: 1.46,1.54$ (total $3 \mathrm{H}$, each d, $\left.J=7 \mathrm{~Hz}, \mathrm{CH}_{3}\right), 2.5-3.7(4 \mathrm{H}$, $\mathrm{m}, \mathrm{SC}_{2} \underline{\mathrm{C}}_{2} \mathrm{~N}$ ), 4.74, 5.68 (total $1 \mathrm{H}$, each q, $J=7 \mathrm{~Hz}, \mathrm{CHCH}_{3}$ ), $6.2-6.4$ $(2 \mathrm{H}, \mathrm{m}, \mathrm{Ar}), 7.1-7.5$ (total $6 \mathrm{H}, \mathrm{m}, \mathrm{Ar}$ ) $8.09,8.27$ (total $1 \mathrm{H}$, each s, NCHO). LR-EI-MS m/z: $275\left(\mathrm{M}^{+}\right)$. HR-EI-MS: Calcd for $\mathrm{C}_{15} \mathrm{H}_{17} \mathrm{NO}_{2} \mathrm{~S}$ : 275.0980. Found: 275.0974.

$N$-(2-Phenylsulfanylethyl)- $N$-(1-thiophen-2-ylethyl)formamide (6b) $(6.96 \mathrm{~g}, 91 \%)$ was obtained from $\mathbf{5 b}(7.00 \mathrm{~g}, 22.8 \mathrm{mmol})$ after purification by column chromatography (AcOEt: hexane $=2: 3$ ) as a pale yellow oil. IR: 1672. ${ }^{1} \mathrm{H}-\mathrm{NMR}: 1.57,1.66$ (total $\left.3 \mathrm{H}, \mathrm{d}, J=7 \mathrm{~Hz}, \mathrm{CH}_{3}\right), 2.7-3.5(4 \mathrm{H}, \mathrm{m}$, $\mathrm{SCH}_{2} \mathrm{CH}_{2} \mathrm{~N}$ ), 4.97, 5.90 (total $\left.1 \mathrm{H}, \mathrm{q}, J=7 \mathrm{~Hz}, \mathrm{CHCH}_{3}\right), 6.9-7.3(8 \mathrm{H}, \mathrm{m}$, Ar), 8.09, 8.32 (total 1H, s, NCHO). LR-EI-MS $m / z$ : $291\left(\mathrm{M}^{+}\right)$. HR-EI-MS: Calcd for $\mathrm{C}_{15} \mathrm{H}_{17} \mathrm{NOS}_{2}: 291.0752$. Found: 291.0786.

$\mathrm{N}$-(1-Benzofuran-2-ylethyl)- $\mathrm{N}$-(2-phenylsulfanylethyl)formamide (6c) $(5.25 \mathrm{~g}, 96 \%)$ was obtained from $5 \mathrm{c}(5.00 \mathrm{~g}, 16.8 \mathrm{mmol})$ after purification by 
column chromatography (AcOEt: hexane $=1: 2$ ) as a pale yellow oil. IR: 1672, 1583. ${ }^{1} \mathrm{H}-\mathrm{NMR}: 1.56,1.63$ (total $3 \mathrm{H}$, each d, $J=7 \mathrm{~Hz}, \mathrm{CH}_{3}$ ), $2.6-3.1$ $\left(2 \mathrm{H}, \mathrm{m}, \mathrm{SC}_{2} \mathrm{CH}_{2} \mathrm{~N}\right), 3.3-3.4\left(2 \mathrm{H}, \mathrm{m}, \mathrm{SCH}_{2} \underline{\mathrm{C}}_{2} \mathrm{~N}\right), 4.86,5.78$ (total $1 \mathrm{H}$, each q, $\left.J=7 \mathrm{~Hz}, \mathrm{CHCH}_{3}\right), 6.56,6.62$ (total $1 \mathrm{H}$, each s, Ar), 7.1-7.6 (9H, m, Ar), 8.15, 8.35 (total $1 \mathrm{H}$, each s, NCHO). LR-EI-MS $m / z: 357\left(\mathrm{M}^{+}\right.$). HR-EIMS: Calcd for $\mathrm{C}_{19} \mathrm{H}_{19} \mathrm{NO}_{2} \mathrm{~S}$ : 325.1134, Found: 325.1127.

$N$-[1-(Benzo[b]thiophen-2-yl)ethyl]- $N$-(2-phenylsulfanylethyl)formamide (6d) $(7.26 \mathrm{~g}, 75 \%)$ was obtained from 2-acetylbenzo[b]thiophene (4d) $(5.00 \mathrm{~g}, 28.4 \mathrm{mmol})$ followed by the formylation of crude resulting amine (5d) after purification by column chromatography (hexane : $\mathrm{AcOEt}=2: 1$ ) as a pale yellow oil. IR: $1672,1583 .{ }^{1} \mathrm{H}-\mathrm{NMR}$ : $1.63,1.72$ (total $3 \mathrm{H}$, each d, $\left.J=7 \mathrm{~Hz}, \mathrm{CH}_{3}\right), 2.8-3.5\left(4 \mathrm{H}, \mathrm{m}, \mathrm{SC}_{2} \mathrm{CH}_{2} \mathrm{~N}-\right), 5.02,5.94$ (total $1 \mathrm{H}$, each q, $\left.J=7 \mathrm{~Hz}, \mathrm{CHCH}_{3}\right), 7.1-8.0(10 \mathrm{H}, \mathrm{m}, \mathrm{Ar}), 8.16,8.37(1 \mathrm{H}$, each s, NCHO). LR-EI-MS $m / z$ : $341\left(\mathrm{M}^{+}\right)$. HR-EI-MS: Calcd for $\mathrm{C}_{19} \mathrm{H}_{19} \mathrm{NOS}_{2}: 341.0908$. Found: 341.0925 .

Oxidation of 6a with $\mathrm{NaIO}_{4}$. Typical Procedure A solution of 6 a $(8.00 \mathrm{~g}, 29.1 \mathrm{mmol})$ and $\mathrm{NaIO}_{4}(9.30 \mathrm{~g}, 43.65 \mathrm{mmol})$ in $\mathrm{MeOH}(200 \mathrm{ml})$ and $\mathrm{H}_{2} \mathrm{O}(50 \mathrm{ml})$ was stirred at room temperature for $1.5 \mathrm{~h}$. After removal of inorganic precipitates by filtration, the filtrate was concentrated in vacuo. The residue was extracted with $\mathrm{CHCl}_{3}$. The product was chromatographed with AcOEt and AcOEt: $\mathrm{MeOH}=9: 1$ to give $7 \mathbf{a}(6.23 \mathrm{~g}, 74 \%)$ and $8 \mathrm{a}(1.67 \mathrm{~g}$, $17 \%)$.

$\mathrm{N}$-(1-Furan-2-ylethyl)- $\mathrm{N}$-(2-phenylsulfinylethyl)formamide (7a) A colorless oil. IR: $1670 .{ }^{1} \mathrm{H}-\mathrm{NMR}: 1.41,1.47,1.56,1.60$ (total $3 \mathrm{H}$, each d, $\left.J=7 \mathrm{~Hz}, \mathrm{CH}_{3}\right), 2.2-4.8\left(4 \mathrm{H}, \mathrm{m}, \mathrm{SC}_{2} \mathrm{C}_{2} \mathrm{~N}\right), 4.76,4.77,5.62,5.72$ (total $1 \mathrm{H}$, each q, $\left.J=7 \mathrm{~Hz}, \mathrm{CHCH}_{3}\right), 6.2-6.4(2 \mathrm{H}, \mathrm{m}, \mathrm{Ar}), 7.3-7.6(6 \mathrm{H}, \mathrm{m}, \mathrm{Ar}-$ $\mathrm{H}), 8.05,8.22,8.23,8.24$ (total $1 \mathrm{H}$, each s, NCHO). LR-EI-MS $m / z: 291$ $\left(\mathrm{M}^{+}\right)$. HR-EI-MS: Calcd for $\mathrm{C}_{15} \mathrm{H}_{17} \mathrm{NO}_{3} \mathrm{~S}: 291.0948$. Found: 291.0930 .

$\boldsymbol{N}$-(1-Furan-2-ylethyl)- $\mathrm{N}$-(2-phenylsulfonylethyl)formamide (8a) A colorless oil. IR: 1670. ${ }^{1} \mathrm{H}-\mathrm{NMR}$ : 1.41, 1.56 (total $3 \mathrm{H}$, each d, $J=7 \mathrm{~Hz}$, $\left.\mathrm{CH}_{3}\right), 2.5-3.6\left(4 \mathrm{H}, \mathrm{m}, \mathrm{SC}_{\mathrm{H}_{2}} \mathrm{C}_{2} \mathrm{~N}\right), 4.77,5.61$ (total $1 \mathrm{H}$, each q, $J=7 \mathrm{~Hz}$, $\mathrm{CHCH}_{3}$ ), 6.2-6.4 (2H, m, Ar), 7.3-7.9 (total 6H, m, Ar), 8.08, 8.19 (total $1 \mathrm{H}$, each s, NCHO). LR-EI-MS $\mathrm{m} / z$ : $307\left(\mathrm{M}^{+}\right)$. HR-EI-MS: Calcd for $\mathrm{C}_{15} \mathrm{H}_{17} \mathrm{NO}_{4} \mathrm{~S}: 307.0860$. Found: 307.0876 .

Oxidation of $\mathbf{6 b} \quad 7 \mathbf{b}(2.00 \mathrm{~g}, 94 \%)$ and $\mathbf{8 b}(76 \mathrm{mg}, 3 \%)$ were obtained from $\mathbf{6 b}(2.00 \mathrm{~g}, 29.1 \mathrm{mmol})$ after purification by column chromatography (AcOEt $:$ hexane $=2: 1$ and AcOEt).

$\mathrm{N}$-(2-Phenylsulfinylethyl)- $\mathrm{N}$-(1-thiophen-2-ylethyl)formamide (7b) An orange oil. IR: 1672. ${ }^{1} \mathrm{H}-\mathrm{NMR}: 1.56,1.59,1.70,1.75$ (total $3 \mathrm{H}$, each d, $\left.J=7 \mathrm{~Hz}, \mathrm{CH}_{3}\right), 2.3-3.8\left(4 \mathrm{H}, \mathrm{m}, \mathrm{SC}_{2} \mathrm{C}_{2} \mathrm{~N}\right), 5.0-6.0$ (total $1 \mathrm{H}, \mathrm{m}$, $\left.\mathrm{CHCH}_{3}\right), 6.9-7.6(8 \mathrm{H}, \mathrm{m}, \mathrm{Ar}), 8.10,8.24,8.32$ (total $\left.1 \mathrm{H}, \mathrm{s}, \mathrm{NCHO}\right)$. LREI-MS: $\mathrm{m} / \mathrm{z}: 323\left(\mathrm{M}^{+}\right)$. HR-EI-MS: Calcd for $\mathrm{C}_{15} \mathrm{H}_{17} \mathrm{NO}_{3} \mathrm{~S}_{2}: 323.0650$. Found: 323.0643 .

$\mathrm{N}$-(2-Phenylsulfonylethyl)- $\mathrm{N}$-(1-thiophen-2-ylethyl)formamide (8b) An orange oil. IR: 1670. ${ }^{1} \mathrm{H}-\mathrm{NMR}: 1.44,1.61$ (total $3 \mathrm{H}$, each d, $J=7 \mathrm{~Hz}$, $\mathrm{CH}_{3}$ ), 2.7-3.6 (total 4H, m, $\mathrm{SC}_{2} \mathrm{C}_{2} \mathrm{~N}$ ), 4.94, 5.80 (total 1H, m, $\mathrm{CHCH}_{3}$ ), $6.8-7.9$ (total $8 \mathrm{H}, \mathrm{m}, \mathrm{Ar}$ ), 8.10, 8.24, 8.32 (total $1 \mathrm{H}, \mathrm{s}, \mathrm{NCHO}$ ). Chemical ionization (CI)-MS $m / z: 308\left(\mathrm{M}^{+}\right)$.

Oxidation of $6 \mathbf{c} 7 \mathbf{c}(3.06 \mathrm{~g}, 97 \%)$ and $8 \mathbf{c}(32 \mathrm{mg}, 1 \%)$ were obtained from $\mathbf{6 c}(3.00 \mathrm{~g}, 9.24 \mathrm{mmol})$ after purification by column chromatography (AcOEt $:$ hexane $=2: 1$ and AcOEt).

$\mathrm{N}$-(1-Benzofuran-2-ylethyl)- $\mathrm{N}$-(2-phenylsulfinylethyl)formamide (7c) Colorless oil. IR: $1670 .{ }^{1} \mathrm{H}-\mathrm{NMR}: 1.55,1.61,1.71,1.74$ (total $3 \mathrm{H}$, each $\mathrm{d}$, $\left.J=7 \mathrm{~Hz}, \quad \mathrm{CH}_{3}\right), \quad 2.6-3.2\left(2 \mathrm{H}, \quad \mathrm{m}, \quad \mathrm{SC}_{2} \mathrm{CH}_{2} \mathrm{~N}\right), \quad 3.3-3.7 \quad(2 \mathrm{H}, \quad \mathrm{m}$, $\mathrm{SCH}_{2} \underline{\mathrm{CH}}_{2} \mathrm{~N}$ ), 4.8-6.7 (total $1 \mathrm{H}, \mathrm{m}, \mathrm{CHCH}_{3}$ ) , 6.64, 6.67, 6.69 (total $1 \mathrm{H}, \mathrm{s}$, Ar), 7.1-7.6 (total 9H, m, Ar), 8.14, 8.31, 8.35 (total 1H, each s, NCHO). LR-EI-MS $m / z$ : $341\left(\mathrm{M}^{+}\right)$. HR-EI-MS: Calcd for $\mathrm{C}_{19} \mathrm{H}_{19} \mathrm{NO}_{3} \mathrm{~S}: 341.1121$. Found: 341.1086.

$\mathrm{N}$-(1-Benzofuran-2-ylethyl)- $\mathrm{N}$-(2-phenylsulfonylethyl)formamide $(\mathbf{8 c})$ A colorless oil. IR: 1672. ${ }^{1} \mathrm{H}-\mathrm{NMR}: 1.52,1.68$ (total $3 \mathrm{H}$, each d, $J=7 \mathrm{~Hz}$, $\left.\mathrm{CH}_{3}\right), 2.7-3.7\left(4 \mathrm{H}, \mathrm{m}, \mathrm{SCH}_{2} \mathrm{CH}_{2} \mathrm{~N}\right), 4.90,5.73$ (total $1 \mathrm{H}$, each q, $J=7 \mathrm{~Hz}$, $\mathrm{CH} \mathrm{CH}_{3}$ ), 6.59, 6.64 (total $1 \mathrm{H}$, each s, Ar), 7.2-7.7 (9H, m, Ar), 8.16, 8.27 (total 1H, each s, NCHO). LR-EI-MS m/z: $357\left(\mathrm{M}^{+}\right)$. HR-EI-MS: Calcd for $\mathrm{C}_{19} \mathrm{H}_{19} \mathrm{NO}_{4} \mathrm{~S}: 357.1020$. Found: 357.1008 .

Oxidation of 6d $N$-[1-(Benzo[b]thiophen-2-yl)ethyl]- $N$-(2-phenylsulfinylethyl)formamide (7d) $(1.55 \mathrm{~g}, 99 \%)$ was obtained from $6 \mathbf{d}(1.50 \mathrm{~g}$, $4.4 \mathrm{mmol})$ after purification by column chromatography $(\mathrm{AcOEt}:$ hexane $=$ $2: 1$ ) as a pale yellow oil. IR: 1655. ${ }^{1} \mathrm{H}-\mathrm{NMR}: 1.63,1.67,1.78$ (total $3 \mathrm{H}$, each d, $\left.J=7 \mathrm{~Hz}, \mathrm{CH}_{3}\right), 2.4-3.9\left(4 \mathrm{H}, \mathrm{m}, \mathrm{SC}_{2} \mathrm{CH}_{2} \mathrm{~N}\right), 5.06,5.95,6.04$ (total $1 \mathrm{H}$, each q, $\left.J=7 \mathrm{~Hz}, \mathrm{CHCH}_{3}\right), 7.1-7.8(10 \mathrm{H}, \mathrm{m}, \mathrm{Ar}$ ), 8.16, 8.29, 8.36 (total $1 \mathrm{H}, \mathrm{s}, \mathrm{NCHO})$. CI-MS $m / z: 358\left(\mathrm{MH}^{+}\right)$.

Pummerer Reaction of Sulfoxide 7b. Typical Procedure i) Method A: TFAA $(2.27 \mathrm{ml}, 16.3 \mathrm{mmol})$ was added to a solution of $7 \mathbf{b}(1.00 \mathrm{~g}$, $3.25 \mathrm{mmol})$ in benzene $(60 \mathrm{ml})$ at room temperature, and the mixture was stirred for $6 \mathrm{~h}$ at the same temperature. The reaction mixture was concentrated in vacuo, and the residue was purified by column chromatography (AcOEt : hexane $=1: 2)$ to give $\mathbf{1 0}(841 \mathrm{mg}, 89 \%)$.

ii) Method B: TFAA ( $2.27 \mathrm{ml}, 16.3 \mathrm{mmol})$ was added to a solution of $7 \mathbf{b}$ $(1.00 \mathrm{~g}, 3.25 \mathrm{mmol})$ in benzene $(60 \mathrm{ml})$ at room temperature. After the mixture was stirred for $0.5 \mathrm{~h}, \mathrm{BF}_{3} \cdot \mathrm{Et}_{2} \mathrm{O}(0.41 \mathrm{ml}, 3.25 \mathrm{mmol})$ was added, and the reaction mixture was further stirred at the same temperature for $1 \mathrm{~h}$. The reaction mixture was diluted with $\mathrm{H}_{2} \mathrm{O}$ and extracted with $\mathrm{CHCl}_{3}$. Purification by column chromatography (AcOEt: hexane $=1: 2$ ) of the product gave $\mathbf{1 0}$ (860 mg, 93\%).

4,5,6,7-Tetrahydro-7-methyl-4-phenylsulfanylthieno[2,3-c]pyridine-6carbaldehyde (10) Colorless oil. IR: 1670. UV: 220 (17700). ${ }^{1} \mathrm{H}-\mathrm{NMR}$ : $1.41,1.47,1.49,1.54$ (total $3 \mathrm{H}$, each d, $\left.J=7 \mathrm{~Hz}, \mathrm{CH}_{3}\right), 3.0-4.7(3 \mathrm{H}, \mathrm{m}, 4-\mathrm{H}$ and $5-\mathrm{H}), 4.85,4.91,5.53,5.60($ total $1 \mathrm{H}, \mathrm{q}, J=7 \mathrm{~Hz}, 7-\mathrm{H}), 6.9-7.6(7 \mathrm{H}, \mathrm{m}$, Ar), 8.03, 8.05, 8.24, 8.39 (total $1 \mathrm{H}$, each s, NCHO). LR-EI-MS $\mathrm{m} / z: 289$ $\left(\mathrm{M}^{+}\right)$. HR-EI-MS: Calcd for $\mathrm{C}_{15} \mathrm{H}_{15} \mathrm{NOS}_{2}: 289.0595$. Found: 289.0635 .

Pummerer Reaction of $7 \mathbf{a}$ The reaction of $7 \mathbf{a}$ with TFAA $(5 \mathrm{~mol}$ eq) under the method A or B conditions described above merely yielded uncharacterable mixtures.

Pummerer Reaction of 7a with Acetic Anhydride A solution of 7a $(700 \mathrm{mg}), \mathrm{Ac}_{2} \mathrm{O}(25 \mathrm{ml})$ and pyridine $(0.5 \mathrm{ml})$ was heated at $80^{\circ} \mathrm{C}$ for $14 \mathrm{~d}$. The reaction mixture was concentrated in vacuo, the product was purified by column chromatography (AcOEt:hexane $=1: 2)$ to give acetic acid 2[formyl-(1-furan-2-ylethyl)amino]-1-phenylsulfanylethyl ester (9) $(685 \mathrm{mg}$, $86 \%$ ) as colorless oil. IR: $1749,1676 .{ }^{1} \mathrm{H}-\mathrm{NMR}: 1.45,1.46,1.54,1.59$ (total $3 \mathrm{H}$, each d, $J=7 \mathrm{~Hz}, \mathrm{CH}_{3}$ ), 2.00, 2.04, 2.06, 2.09 (total 3H, each s, OAc), $3.2-3.7\left(2 \mathrm{H}, \mathrm{m}, \mathrm{NC}_{2} \mathrm{CHS}\right), 4.69,4.70,5.58,5.64$ (total $1 \mathrm{H}$, each q, $J=7 \mathrm{~Hz}, \mathrm{CHCH}_{3}$ ), 5.05, 5.85, 6.10 (total $1 \mathrm{H}$, each dd, $J=3,9 \mathrm{~Hz}$, $\left.\mathrm{NCH}_{2} \mathrm{C} \underline{\mathrm{HS}}\right), 6.2-6.3(2 \mathrm{H}, \mathrm{m}, \mathrm{Ar}), 7.3-7.5(6 \mathrm{H}, \mathrm{m}, \mathrm{Ar}-\mathrm{H}), 8.00,8.01,8.23$, 8.27 (total $1 \mathrm{H}$, each s, NCHO). LR-EI-MS $m / z$ : $334\left(\mathrm{M}^{+}\right)$. HR-FAB-MS: Calcd for $\mathrm{C}_{17} \mathrm{H}_{20} \mathrm{NO}_{4} \mathrm{~S}$ : 334.1116 . Found: 334.1113 .

Treatment of 9 with $\mathbf{B F}_{3} \cdot \mathbf{E t}_{2} \mathbf{O}$ A solution of $\mathbf{9}(333 \mathrm{mg})$ and $\mathrm{BF}_{3} \cdot \mathrm{Et}_{2} \mathrm{O}$ $(0.13 \mathrm{ml})$ in benzene $(16 \mathrm{ml})$ was allowed to react at $\mathrm{rt}$ for $17 \mathrm{~h}$ under stirring. The products obtained as dark reddish oil gave many spots on TLC.

Reductive Desulfurization of $\mathbf{1 0} \mathrm{NaBH}_{4}(610 \mathrm{mg}, 18.1 \mathrm{mmol})$ was added in small portions to a stirred solution of $\mathbf{1 0}(509 \mathrm{mg}, 1.76 \mathrm{mmol})$ and $\mathrm{NiCl}_{2} \cdot 6 \mathrm{H}_{2} \mathrm{O}(1.26 \mathrm{~g}, 3.5 \mathrm{mmol})$ in EtOH $(100 \mathrm{ml})$ under ice-cooling. The mixture was stirred at room temperature for a further $30 \mathrm{~min}$. To the reaction mixture $\mathrm{H}_{2} \mathrm{O}(10 \mathrm{ml})$ was added and filtered. The filtrate was diluted with $\mathrm{H}_{2} \mathrm{O}$ and acidified by $5 \% \mathrm{HCl}$ solution, and the mixture was extracted with $\mathrm{CHCl}_{3}$. The residue was purified by flash chromatography $(\mathrm{AcOEt}:$ hexane $=$ $1: 1)$ to give 4,5,6,7-tetrahydro-7-methylthieno[2,3-c]pyridine-6-carbaldehyde (11) $(194 \mathrm{mg}, 61 \%)$ as a colorless oil. IR: $1672 .{ }^{1} \mathrm{H}-\mathrm{NMR}: 1.50,1.59$ (total $\left.3 \mathrm{H}, \mathrm{d}, J=7 \mathrm{~Hz}, \mathrm{CH}_{3}\right), 2.7-4.6(4 \mathrm{H}, \mathrm{m}, 4-\mathrm{H}$ and $5-\mathrm{H}), 4.91,5.57$ (total $1 \mathrm{H}, \mathrm{q}, J=7 \mathrm{~Hz}, 7-\mathrm{H}), 6.7-7.2(2 \mathrm{H}, \mathrm{m}, \mathrm{Ar}), 8.31,8.35$ (total 1H, s, NCHO). LR-EI-MS m/z: $181\left(\mathrm{M}^{+}\right)$. HR-EI-MS: Calcd for $\mathrm{C}_{9} \mathrm{H}_{11} \mathrm{NOS}: 181.0561$. Found: 181.0570 .

Hydrolysis of 11 A solution of $11(600 \mathrm{mg}, 3.4 \mathrm{mmol})$ in EtOH $(10 \mathrm{ml})-10 \% \mathrm{NaOH}(10 \mathrm{ml})$ was refluxed for $16 \mathrm{~h}$. The reaction mixture was diluted with $\mathrm{H}_{2} \mathrm{O}$ and the mixture was extracted with $\mathrm{CHCl}_{3}$. The product was purified by column chromatography $\left(\mathrm{CHCl}_{3}: \mathrm{MeOH}=9: 1\right)$ to give 4,5,6,7-tetrahydro-7-methylthieno[2,3-c]pyridine (1b) $\left.{ }^{15}\right)(414 \mathrm{mg}, 79 \%)$ as a pale yellow oil (lit $(\mathrm{HCl}$ salt: Colorless needles recrystallized from EtOH-Et ${ }_{2} \mathrm{O}, \mathrm{mp} 230-234^{\circ} \mathrm{C}$ ). IR: no carbonyl absorption. ${ }^{1} \mathrm{H}-\mathrm{NMR}: 1.45$ $\left(3 \mathrm{H}, \mathrm{d}, J=7 \mathrm{~Hz}, \mathrm{CH}_{3}\right), 2.55-2.75,2.80-3.45$ (each $2 \mathrm{H}, \mathrm{m}, 4-\mathrm{H}$ and $5-\mathrm{H}$ ), $4.13(1 \mathrm{H}, \mathrm{q}, J=7 \mathrm{~Hz}, 7-\mathrm{H}), 6.77(1 \mathrm{H}, \mathrm{d}, J=5 \mathrm{~Hz}, 2-\mathrm{H}), 7.10(1 \mathrm{H}, \mathrm{dd}, J=1$, $5 \mathrm{~Hz}, 3-\mathrm{H}) .{ }^{13} \mathrm{C}-\mathrm{NMR}: 23.5\left(\mathrm{CH}_{3}\right), 26.8(\mathrm{C} 4), 42.9$ (C5), 50.6 (C7), 121.9 (C2), 127.5 (C3), 133.8 (C7a), 140.4 (C3a). LR-EI-MS m/z: $153\left(\mathrm{M}^{+}\right)$. HREI-MS: Calcd for $\mathrm{C}_{8} \mathrm{H}_{11}$ NS: 153.0612. Found: 153.0600 .

Pummerer Reaction of 7c i) Method A: TFAA $(4.11 \mathrm{ml}, 22 \mathrm{mmol})$ was added to a solution of $7 \mathbf{c}(1.50 \mathrm{~g}, 4.39 \mathrm{mmol})$ in dry benzene $(75 \mathrm{ml})$ at room temperature, and the mixtrue was stirred for $1.5 \mathrm{~h}$ at the same temperature. The reaction mixture was diluted with $\mathrm{H}_{2} \mathrm{O}$ and the mixture was extracted with $\mathrm{CHCl}_{3}$. The residue was purified by column chromatography (AcOEt: hexane $=1: 2)$ to give 12a $(516 \mathrm{mg}, 36 \%), \mathbf{1 3}(496 \mathrm{mg}, 35 \%)$, and $\mathbf{1 4}$ (230 mg, 16\%).

ii) Method B: TFAA $(1.02 \mathrm{ml}, 7.33 \mathrm{mmol})$ was added to a solution of $7 \mathbf{c}$ $(500 \mathrm{mg}, 1.47 \mathrm{mmol})$ in benzene $(30 \mathrm{ml})$ at room temperature. After the mixture was stirred for $0.5 \mathrm{~h}, \mathrm{BF}_{3} \cdot \mathrm{Et}_{2} \mathrm{O}(0.56 \mathrm{ml}, 4.40 \mathrm{mmol})$ was added, and the reaction mixture was further stirred at the same temperature for $0.5 \mathrm{~h}$. The reaction mixture was diluted with $\mathrm{H}_{2} \mathrm{O}$ and extracted with $\mathrm{CHCl}_{3}$. Purification by column chromatography (AcOEt : hexane $=2: 3$ ) of the product gave 12a (143 mg, 30\%), 13 (187 mg, 39\%) and 14 (127 mg, 27\%).

1,2,3,4-Tetrahydro-1-methyl-4-phenylsulfanylbenzo[4,5]furo[2,3-c]- 
pyridine-2-carbaldehyde (12a) Colorless needles recrystallized from AcOEt-hexane, mp: $184-186^{\circ} \mathrm{C}$. UV: 248 (17000), 281 (3800). IR: 1668, 1662. ${ }^{1} \mathrm{H}$-NMR: 1.50, 1.56 (total $3 \mathrm{H}$, each d, $J=7 \mathrm{~Hz}, \mathrm{CH}_{3}$ ), 3.25, 3.77 (total $1 \mathrm{H}$, dd, $J=3,11 \mathrm{~Hz}, 3-\mathrm{H}), 3.68,4.75$ (total $1 \mathrm{H}, \mathrm{dd}, J=1,14 \mathrm{~Hz}, 3-\mathrm{H}), 4.4-$ 4.6 (total $1 \mathrm{H}, \mathrm{m}, 4-\mathrm{H}), 4.83,5.54$ (total $1 \mathrm{H}$, each d, $J=7 \mathrm{~Hz}, 1-\mathrm{H}$ ), $7.2-7.7$ $(9 \mathrm{H}, \mathrm{m}, \mathrm{Ar}), 8.13,8.42$ (total $1 \mathrm{H}$, each $\mathrm{s}$, NCHO). LR-EI-MS $\mathrm{m} / \mathrm{z}: 323$ $\left(\mathrm{M}^{+}\right)$. HR-EI-MS: Calcd for $\mathrm{C}_{19} \mathrm{H}_{17} \mathrm{NO}_{2} \mathrm{~S}$ : 323.0981, Found: 323.0980 . Anal. Calcd for $\mathrm{C}_{19} \mathrm{H}_{17} \mathrm{NO}_{2} \mathrm{~S}$ : C, 70.56; H, 5.30; N, 4.33. Found: C, 70.16; $\mathrm{H}, 5.26 ; \mathrm{N}, 4.12$.

$\left(1 R^{*}, 9 S^{*}, 10 S^{*}, 18 R^{*}\right)$-19-Formyl-18-methyl-19-aza-2-oxa-11-thiapentacyclo $\left[8.7 .3 .0^{1,9} \cdot 0^{3,8} \cdot 0^{12,17}\right]$ icosa-3,5,7,12,14,16-hexaene (13) Colorless plates recrystallized from $\mathrm{CHCl}_{3}-\mathrm{MeOH}, \mathrm{mp}: 258-260^{\circ} \mathrm{C}$. UV: 261 (8000), 281 (4300), 290 (3800). IR: 1662, 1655, 1597. ${ }^{1} \mathrm{H}-\mathrm{NMR}: 1.35,1.39$ (total $3 \mathrm{H}$, each d, $\left.J=7 \mathrm{~Hz}, \mathrm{CH}_{3}\right), 3.6-3.8(3 \mathrm{H}, \mathrm{m}, 10-\mathrm{H}$ and $20-\mathrm{H}), 4.23$, 4.43 (total $1 \mathrm{H}$, each q, $J=7 \mathrm{~Hz}, 18-\mathrm{H}$ ), 4.61, 4.65 (total $1 \mathrm{H}$, each s, 9-H), 6.8-7.5 (8H, m, Ar), 8.34, 8.35 (total $1 \mathrm{H}$, each s, NCHO). LR-EI-MS $m / z$ : $323\left(\mathrm{M}^{+}\right)$. HR-EI-MS: Calcd for $\mathrm{C}_{19} \mathrm{H}_{17} \mathrm{NO}_{2} \mathrm{~S}: 323.0972$, Found: 323.0978. Anal. Calcd for $\mathrm{C}_{19} \mathrm{H}_{17} \mathrm{NO}_{2} \mathrm{~S}$ : C, 70.56; H, 5.30; N, 4.33. Found: C, 70.27; $\mathrm{H}, 5.32 ; \mathrm{N}, 4.15$

$\left(1 S^{*}, 9 R^{*}, 10 R^{*}, 18 R^{*}\right)$-19-Formyl-18-methyl-19-aza-2-oxa-11-thiapentacyclo $\left[8.7 .3 .0^{1,9} \cdot 0^{3,8} \cdot 0^{12,17}\right]$ icosa-3,5,7,12,14,16-hexaene (14) Colorless needles recrystallized from $\mathrm{CHCl}_{3}-\mathrm{MeOH}, \mathrm{mp}: 245-248^{\circ} \mathrm{C}$. UV: 260 (3900), 281 (2100), 290 (1900). IR: 1649, 1595. ${ }^{1} \mathrm{H}-\mathrm{NMR}: 1.41,1.43$ (total $3 \mathrm{H}$, each d, $\left.J=6 \mathrm{~Hz}, \mathrm{CH}_{3}\right), 3.4-4.2(3 \mathrm{H}, \mathrm{m}, 10-\mathrm{H}$ and $20-\mathrm{H}), 3.89,3.99$ (total $1 \mathrm{H}$, each q, $J=6 \mathrm{~Hz}, 18-\mathrm{H}$ ), 4.54, 4.56 (total $1 \mathrm{H}$, each s, 9-H), $6.8-$ $7.4(8 \mathrm{H}, \mathrm{m}, \mathrm{Ar}), 8.29,8.36$ (total $1 \mathrm{H}$, each s, NCHO). LR-EI-MS $\mathrm{m} / z: 323$ $\left(\mathrm{M}^{+}\right)$. HR-EI-MS: Calcd for $\mathrm{C}_{18} \mathrm{H}_{17} \mathrm{NO}_{2} \mathrm{~S}$ : 323.0952, Found: 323.0979 . Anal. Calcd for $\mathrm{C}_{19} \mathrm{H}_{17} \mathrm{NO}_{2} \mathrm{~S}$ : C, 70.56; H, 5.30; N, 4.33. Found: C, 70.26; H, $5.29 ; \mathrm{N}, 4.12$.

Pummerer Reaction of 7d i) Method A: 12b (893 mg, 94\%) was obtained from $7 \mathbf{d}(1.00 \mathrm{~g}, 2.81 \mathrm{mmol})$ after purification by column chromatography (AcOEt : hexane $=1: 2$ ).

ii) Method B: 12b (175 mg, 99\%) was obtained from 7d (187 mg, $0.523 \mathrm{mmol})$.

1,2,3,4-Tetrahydro-1-methylbenzo[4,5]thieno[2,3-c]pyridine-2-carbaldehyde (12b) Colorless needles recrystallized from AcOEt-hexane, mp: 173-175 ${ }^{\circ}$ C. IR: 1672, 1664. UV: 227 (34300), 259 (13200), 297 (3400). ${ }^{1} \mathrm{H}$-NMR: $1.35,1.54,1.62$ (total $3 \mathrm{H}$, each d, $\left.J=6 \mathrm{~Hz}, \mathrm{CH}_{3}\right), 3.3-4.8$ $(3 \mathrm{H}, \mathrm{m}, 3-\mathrm{H}$ and $4-\mathrm{H}), 4.86,5.00,5.53,5.60$ (total $1 \mathrm{H}$, each q, $J=6 \mathrm{~Hz}, 1-$ H), 7.3-7.9 (9H, m, Ar), 8.09, 8.17, 8.37, 8.47 (total $1 \mathrm{H}, \mathrm{s}, \mathrm{NCHO})$. LREI-MS $m / z: 339\left(\mathrm{M}^{+}\right)$. HR-EI-MS: Calcd for $\mathrm{C}_{19} \mathrm{H}_{17} \mathrm{NOS}_{2}$ : 339.0749, Found: 339.0748. Anal. Calcd for $\mathrm{C}_{19} \mathrm{H}_{17} \mathrm{NOS}_{2}$ : C, 67.22; H, 5.05; N, 4.13. Found: C, 66.97; H, 5.02; N, 3.94 .

Reductive Desulfurization of 12a. Typical Experiment $\mathrm{NaBH}_{4}$ $(610 \mathrm{mg}, 18.8 \mathrm{mmol})$ was added in small portions to a stirred solution of 12a $(607 \mathrm{mg}, 1.88 \mathrm{mmol})$ and $\mathrm{NiCl}_{2} \cdot 6 \mathrm{H}_{2} \mathrm{O}(1.38 \mathrm{~g}, 6.6 \mathrm{mmol})$ in $\mathrm{EtOH}(100 \mathrm{ml})$ under ice-cooling. The mixture was stirred at room temperature for a further $0.5 \mathrm{~h}$. To the reaction mixture $\mathrm{H}_{2} \mathrm{O}(10 \mathrm{ml})$ was added and filtered. The filtrate was diluted with $\mathrm{H}_{2} \mathrm{O}$ and acidified by $5 \% \mathrm{HCl}$ solution, and the mixture was extracted with $\mathrm{CHCl}_{3}$. The residue was purified by flash chromatography (AcOEt : hexane $=2: 3$ ) to give 1,2,3,4-tetrahydro-1-methylbenzo[4,5]furo[2,3-c]pyridine-2-carbaldehyde (15a) $(311 \mathrm{mg}, 77 \%)$ as colorless needles recrystallized from AcOEt-hexane, mp $258-260^{\circ} \mathrm{C}$. IR: $1662,1627$. UV: 283 (3600), 277 (4200), 250 (16100). ${ }^{1} \mathrm{H}-\mathrm{NMR}: 1.53,1.60$ (3H, each d, $\left.J=6 \mathrm{~Hz}, \mathrm{CH}_{3}\right), 2.7-4.7(4 \mathrm{H}, \mathrm{m}, 3-\mathrm{H}$ and $4-\mathrm{H}), 4.48,5.52$ (total $1 \mathrm{H}$, each q, $J=7 \mathrm{~Hz}, 1-\mathrm{H}$ ), 7.2-7.5 (4H, m, Ar), 8.19, 8.34 (total 1H, s, NCHO). LR-EIMS $m / z$ : $323\left(\mathrm{M}^{+}\right)$. HR-EI-MS: Calcd for $\mathrm{C}_{13} \mathrm{H}_{13} \mathrm{NO}_{2}$ : 323.0982, Found: 323.0980 .

1,2,3,4-Tetrahydro-1-methylbenzo[4,5]thieno[2,3-c]pyridine-2-carbaldehyde (15b) (383 mg, 71\%) was obtained from 12b (796 mg, $2.35 \mathrm{mmol})$ after purification by column chromatography (benzene : acetone $=15: 2$ ) as a colorless oil. IR: 1670, 1654. UV: 229 (23900), 258 (6800), 287 (2100), 297 (1600). ${ }^{1} \mathrm{H}-\mathrm{NMR}: 1.56,1.65$ (total $\left.3 \mathrm{H}, \mathrm{d}, J=7 \mathrm{~Hz}, \mathrm{CH}_{3}\right), 2.7-4.7(4 \mathrm{H}, \mathrm{m}, 3-$ $\mathrm{H}$ and 4-H), 5.9-6.0 $(1 \mathrm{H}, \mathrm{m}, 1-\mathrm{H}), 7.2-7.8(4 \mathrm{H}, \mathrm{m}, \mathrm{Ar}), 8.20,8.35$ (total $1 \mathrm{H}, \quad \mathrm{s}$, NCHO). LR-EI-MS m/z: $231\left(\mathrm{M}^{+}\right)$. HR-EI-MS: Calcd for $\mathrm{C}_{13} \mathrm{H}_{13} \mathrm{NOS}$ : 231.0718. Found: 231.0700 .

Hydrolysis of 15a. Typical Experiment A solution of 15a $(201 \mathrm{mg}$, $1.08 \mathrm{mmol})$ in EtOH $(20 \mathrm{ml})-10 \% \mathrm{NaOH}(10 \mathrm{ml})$ was refluxed for $1.5 \mathrm{~h}$. The reaction mixture was diluted with $\mathrm{H}_{2} \mathrm{O}$ and the mixture was extracted with $\mathrm{CHCl}_{3}$. The product was purified by column chromatography (AcOEt) to give 1,2,3,4-tetrahydro-1-methylbenzo[4,5]furo[2,3-c]pyridine (2a) (171 mg, $98 \%$ ) as a colorless oil. IR: no carbonyl absorption. UV: 244 (13000), 273 (2600), 280 (2200). ${ }^{1} \mathrm{H}-\mathrm{NMR}: 1.46\left(3 \mathrm{H}, \mathrm{d}, J=7 \mathrm{~Hz}, \mathrm{CH}_{3}\right), 2.5-3.3(4 \mathrm{H}, \mathrm{m}$, $3-\mathrm{H}$ and $4-\mathrm{H}), 4.0-4.1(1 \mathrm{H}, \mathrm{m}, 1-\mathrm{H}), 7.2-7.25(2 \mathrm{H}, \mathrm{m}, \mathrm{Ar}-\mathrm{H}), 7.4-7.42$
$(2 \mathrm{H}, \mathrm{m}, \mathrm{Ar}-\mathrm{H}) .{ }^{13} \mathrm{C}-\mathrm{NMR}: 19.2\left(\mathrm{CH}_{3}\right), 22.8(\mathrm{C} 4), 42.0(\mathrm{C} 3), 48.4(\mathrm{C} 1)$, 111.0 (C8), 111.1 (C4a), 118.6 (C5), 122.3 (C6), 123.3 (C7), 128.3 (C4b), 154.1 (C8a), 156.0 (C9a). LR-EI-MS m/z: $187\left(\mathrm{M}^{+}\right)$. HR-EI-MS: Calcd for $\mathrm{C}_{12} \mathrm{H}_{13} \mathrm{NO}$ : 187.1000 . Found: 187.0997 .

1,2,3,4-Tetrahydro-1-methylbenzo[4,5]thieno[2,3-c]pyridine (2b) (86 mg, $83 \%)$ was obtained from $17 \mathbf{b}(118 \mathrm{mg}, 0.51 \mathrm{mmol})$ after purification by column chromatography (AcOEt: $\mathrm{MeOH}=6: 1$ and $4: 1)$ as a colorless oil $\left(\right.$ lit $^{16)}: \mathrm{HCl}$ salt $\left.\mathrm{mp}: 256-258^{\circ} \mathrm{C}\right)\left(\operatorname{lit}^{17)}:(S)-1,2,3,4\right.$-tetrahydro-1-methylbenzo[4,5]thieno[2,3-c]pyridine $\mathrm{HCl}$ salt mp: $\left.240-242{ }^{\circ} \mathrm{C}\right)$. IR: no carbonyl absorption. UV: 227 (22500), 265 (6400), 298 (1700). ${ }^{1} \mathrm{H}-\mathrm{NMR}: 1.50(3 \mathrm{H}$, d, $\left.J=7 \mathrm{~Hz}, \mathrm{CH}_{3}\right), 2.7-2.8(2 \mathrm{H}, \mathrm{m}, 4-\mathrm{H}), 3.11(1 \mathrm{H}$, ddd, $J=14,13,7 \mathrm{~Hz}, 3-$ H), $3.44(1 \mathrm{H}, \mathrm{td}, J=13,5 \mathrm{~Hz}, 3-\mathrm{H}), 4.23(1 \mathrm{H}, \mathrm{qt}, J=7,2 \mathrm{~Hz}, 1-\mathrm{H}), 7.28(1 \mathrm{H}$, ddd, $J=7.6,7.3,1 \mathrm{~Hz}, 6-\mathrm{H}$ or $7-\mathrm{H}), 7.35(1 \mathrm{H}$, ddd, $J=7.6,7.3,1 \mathrm{~Hz}, 6-\mathrm{H}$ or $7-\mathrm{H}), 7.58(1 \mathrm{H}, \mathrm{d}, J=7 \mathrm{~Hz}, 8-\mathrm{H}), 7.79(1 \mathrm{H}, \mathrm{d}, J=7 \mathrm{~Hz}, 5-\mathrm{H}) .{ }^{13} \mathrm{C}-\mathrm{NMR}: 23.1$ $\left(\mathrm{CH}_{3}\right), 25.0(\mathrm{C} 4), 42.3(\mathrm{C} 3), 50.6(\mathrm{C} 1), 120.7$ (C8), 122.4 (C5), 124.0 (C6 and C7), 128.2 (C4a), 138.3 (C9a), 139.5 (C8b), 141.6 (C4b). LR-EI-MS $m / z: 204\left(\mathrm{M}^{+}\right)$. HR-EI-MS: Calcd for $\mathrm{C}_{12} \mathrm{H}_{13} \mathrm{NS}:$ 204.0835. Found: 204.0847 .

Hydrolysis of 13. Typical Prcedure A solution of $13(200 \mathrm{mg})$ in EtOH $(30 \mathrm{ml})$ and $10 \% \mathrm{NaOH}(10 \mathrm{ml})$ was heated under reflux for $1.5 \mathrm{~h}$. The reaction mixture was diluted with $\mathrm{H}_{2} \mathrm{O}$ and the mixture was extracted with $\mathrm{CHCl}_{3}$. Recrystallyzation of the product from AcOEt-hexane gave $\left(1 R^{*}, 9 S^{*}, 10 S^{*}, 18 R^{*}\right)$-18-methyl-19-aza-2-oxa-11-thiapentacyclo$\left[8.7 .3 .0^{1,9} \cdot 0^{3,8} \cdot 0^{12,17}\right.$ ]icosa- $3,5,7,12,14,16$-hexaene (16) $(175 \mathrm{mg}, 96 \%)$ as colorless plates, mp: $139-142^{\circ} \mathrm{C}$. UV: 261 (7900), 281 (4400), 290 (3700). IR: 1597. LR-EI-MS m/z: $295\left(\mathrm{M}^{+}\right)$. HR-EI-MS: Calcd for $\mathrm{C}_{18} \mathrm{H}_{17} \mathrm{NOS}$ : 295.1071. Found: 295.1031 .

$\left(1 S^{*}, 9 R^{*}, 10 R^{*}, 18 R^{*}\right)$-18-Methyl-19-aza-2-oxa-11-thiapentacyclo$\left[8.7 .3 .0^{1,9} .0^{3,8} .0^{12,17}\right]$ icosa-3,5,7,12,14,16-hexaene (17) $(28 \mathrm{mg}, 85 \%)$ was obtained from $14(36 \mathrm{mg})$ as colorless needles recrystallized from AcOEthexane, mp: $169-170^{\circ} \mathrm{C}$. IR: 1597, 1479. UV: 260 (6600), 282 (3700), 289 (3200). LR-EI-MS $m / z$ : $295\left(\mathrm{M}^{+}\right)$. HR-EI-MS: Calcd for $\mathrm{C}_{18} \mathrm{H}_{17} \mathrm{NOS}$ : 295.1068. Found: 295.1032.

Pummerer Reaction of 7c with Acetic Anhydride A solution of 7c $(702 \mathrm{mg}, 2.06 \mathrm{mmol})$ in $\mathrm{Ac}_{2} \mathrm{O}(10 \mathrm{ml})$ was allowed to react at $80^{\circ} \mathrm{C}$ for $9 \mathrm{~d}$. Column chromatography of the product (AcOEt/hexane 1:2) gave acetic acid 2-[(1-benzofuran-2-ylethyl)formylamino]-1-phenylsulfanylethyl ester (18) $(691 \mathrm{mg}, 87 \%)$ as colorless oil. IR: $1749,1676 .{ }^{1} \mathrm{H}-\mathrm{NMR}: 1.56,1.57$, $1.66,1.69$ (total $3 \mathrm{H}$, each d, $J=7 \mathrm{~Hz}, \mathrm{CH}_{3}$ ) $, 1.82,2.02,2.03,2.07$ (total $3 \mathrm{H}$, each s, $\left.\mathrm{OCOCH}_{3}\right), 3.3-3.8\left(2 \mathrm{H}, \mathrm{m}, \mathrm{NCH}_{2} \mathrm{CHS}\right), 5.46,6.01,6.26,6.28$ (total $1 \mathrm{H}$, each dd, $\left.J=4,9 \mathrm{~Hz}, \mathrm{NCH}_{2} \mathrm{CHS}\right), 4.79-4.87,5.67-5.77(1 \mathrm{H}, \mathrm{m}$, $\left.\mathrm{CHCH}_{3}\right), 6.5-6.6(1 \mathrm{H}, \mathrm{m}, \mathrm{Ar}), 7.1-7.6(9 \mathrm{H}, \mathrm{m}, \mathrm{Ar}-\mathrm{H}), 8.06,8.09,8.32$, 8.36 (total $1 \mathrm{H}$, each s, $\mathrm{NCHO}$ ).

Reaction of 20 with $\mathbf{B F}_{3} \cdot \mathbf{E t}_{2} \mathbf{O}$ A solution of $\mathbf{1 8}(279 \mathrm{mg}, 0.72 \mathrm{mmol})$ in benzene $(15 \mathrm{ml})$ was treated with $\mathrm{BF}_{3} \cdot \mathrm{Et}_{2} \mathrm{O}(0.27 \mathrm{ml}, 2.18 \mathrm{mmol})$ at room temperature for $7 \mathrm{~d}$. Column chromatography of the product (AcOEt: hexane $=2: 3)$ gave 12a $(78 \mathrm{mg}, 33 \%), \mathbf{1 3}(76 \mathrm{mg}, 32 \%)$, and $\mathbf{1 4}(76 \mathrm{mg}$, $32 \%)$.

Treatment of 12a under Acidic Conditions i) A solution of 12a $(302 \mathrm{mg}, 0.93 \mathrm{mmol})$, TFAA $(0.71 \mathrm{ml}, 5.11 \mathrm{mmol})$ in benzene $(20 \mathrm{ml})$ was treated with $\mathrm{BF}_{3} \cdot \mathrm{Et}_{2} \mathrm{O}(0.39 \mathrm{ml}, 3.06 \mathrm{mmol})$ at room temperature for $17 \mathrm{~h}$. Column chromatography of the residue (AcOEt: hexane=1:1) recovered the starting material 12a (295 mg, 98\%).

ii) A solution of 12a ( $300 \mathrm{mg}, 0.93 \mathrm{mmol})$ TFAA $(0.64 \mathrm{ml}, 4.65 \mathrm{mmol})$ in benzene $(20 \mathrm{ml})$ was treated with TFA $(106 \mathrm{mg}, 0.93 \mathrm{mmol})$ at room temperature for $18 \mathrm{~h}$. After treatment in the same manner as described above, recovered the starting material 12a (300 mg, 100\%).

iii) Treatment of 12a $(300 \mathrm{mg}, 0.93 \mathrm{mmol})$ in benzene $(20 \mathrm{ml})$ with TFAA $(0.64 \mathrm{ml}, 4.65 \mathrm{mmol}), \mathrm{BF}_{3} \cdot \mathrm{Et}_{2} \mathrm{O}(0.39 \mathrm{ml}, 3.06 \mathrm{mmol})$ and TFA $(106 \mathrm{mg}$, $0.93 \mathrm{mmol})$ at room temperature for $18 \mathrm{~h}$ recovered $12 \mathrm{a}(300 \mathrm{mg}, 100 \%)$.

iv) Treatment of 12a $(300 \mathrm{mg}, 0.93 \mathrm{mmol})$ in benzene $(20 \mathrm{ml})$ with acetic anhydride $(0.46 \mathrm{ml}, 4.65 \mathrm{mmol})$ and $p$ - TsOH $(160 \mathrm{mg}, 0.93 \mathrm{mmol})$ at $80^{\circ} \mathrm{C}$ for $18 \mathrm{~h}$ recovered 12a $(300 \mathrm{mg}, 100 \%)$.

\section{References}

1) Saitoh T., Shikiya K., Horiguchi Y., Sano T., Chem. Pharm. Bull., 51, 667-672 (2003).

2) Shinohara T., Toda J., Sano T., Chem. Pharm. Bull., 45, 813-819 (1997).

3) Shinohara T., Takeda A., Toda J., Terasawa N., Sano T., Heterocycles, 46, 555-565 (1997).

4) Shinohara T., Takeda A., Toda J., Ueda Y., Kohno M., Sano T., Chem. Pharm. Bull., 46, 918-927 (1998).

5) Shinohara T., Takeda A., Toda J., Sano T., Chem. Pharm. Bull., 46, 
$430-433$ (1998).

6) Toda J., Matsumoto S., Saitoh T., Sano T., Chem. Pharm. Bull., 48, 91-98 (2000).

7) Toda J., Sakagami M., Sano T., Chem. Pharm. Bull., 47, 1269-1275 (1999).

8) Toda J., Ichikawa T., Saithoh T., Horiguchi Y., Sano T., Heterocycles, 53, 2009-2018 (2000).

9) Toda J., Sakagami M., Goan Y., Simakata M., Saitoh T., Horiguchi Y., Sano T., Chem. Pharm. Bull., 48, 1854-1861 (2000).

10) Sano T., Trends Heterocyclic Chem., 7, 117-142 (2001).

11) Ota S., Kono M., Makino Y., Tachikawa O., Hirobe M., Biomed. Res., 8, 453-456 (1987).
12) Tasaki Y., Makino Y., Ohta S., Hirobe M., J. Neurochem., 57, 19401943 (1991).

13) Yamakawa T., Kotake, Y., Fujitani M., Shintani H., Makino Y., Ohta S., Neurosci. Lett., 276, 68-70 (1999).

14) Abe K., Taguchi K., Wasai T., Ren J., Utsunomiya I., Shinohara T., Miyatake T., Sano T., Brain Res. Bull., 56, 55-60 (2001).

15) Gray N. M., Cheng B. K., Mick S. J., Lair C. M., Contreras P. C., J. Med. Chem., 32, 1242-1258 (1989).

16) Clarke K., Hughes C. G., Humphries A. J., Scrowston R. M., J. Org. Chem. (C), 1970, 1013-1016 (1970).

17) Qais N., Nakao N., Hashigaki Y., Takeuchi Y., Yamato M., Chem. Pharm. Bull., 39, 3338-3340 (1991). 